4

5

\title{
High-resolution X-ray imaging and analysis of coatings on and in wood
}

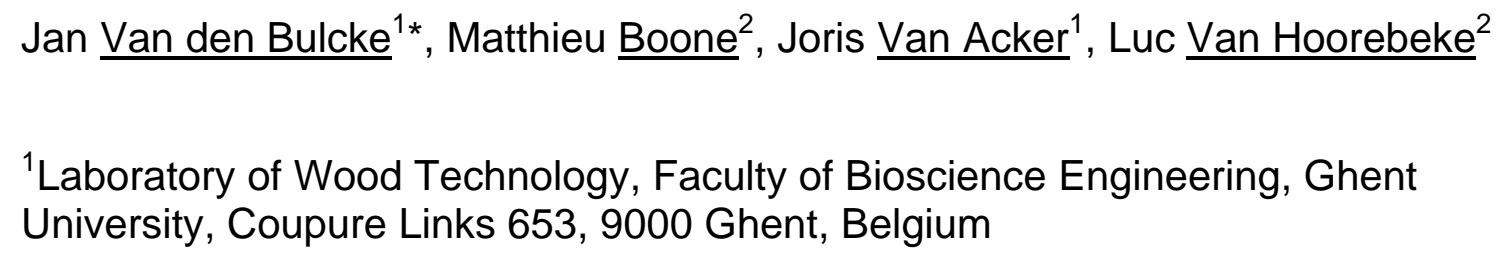

${ }^{2}$ Department for Subatomic and Radiation Physics, Faculty of Sciences, Ghent University, Proeftuinstraat 86, 9000 Gent, Belgium

*e-mail (corresponding author): Jan.VandenBulcke@UGent.be;

postal address: $\quad$ Laboratory of Wood Technology

Coupure links 653

9000 Ghent

Belgium

tel: $+32(0) 92646124$

fax: +32 (0)9264 6233

Cite as:

Van den Bulcke, J., Boone, M., Van Acker, J., Van Hoorebeke, L. High-resolution Xray imaging and analysis of coatings on and in wood. Journal of Coatings Technology and Research. In press.

DOI: 10.1007/s11998-009-9182-4 


\begin{abstract}
Wood coatings are widely used for aesthetic and protective reasons. Assessment of coating performance during service life is crucial in order to establish a knowledge database for product optimization. A vast amount of techniques is available for analysis of a coating's behavior of which micro-imaging is an important tool. In addition to standard microscopy techniques, high-resolution X-ray tomography is presented as a modality offering non-destructive visualization of a coating and the substrate applied on. Combined with analysis of the 3D volumetric data, surface roughness, structure and thickness of the coating layer, penetration depth and related mechanical anchoring can be studied in relation with the underlying substrate. To provide a clear illustration of the possibilities and limitations of this technique, both an opaque solvent-borne and an opaque water-borne coating applied on two different wood types were scanned and analyzed. Clearly, three-dimensional X-ray imaging at high resolution produces valuable information merely by visualization. Moreover by proper analysis quantitative data is obtained taking into account the limitations of $X$ ray computed tomography and of automated image processing.
\end{abstract}

Keywords: 3D; X-ray CT; coating; wood 


\section{Introduction}

In spite of the use of synthetic alternatives, the importance of wood as an industrial material cannot be underestimated. Although its undeniable advantage as a renewable material with unique properties, wood has the disadvantage that due to its biological nature, it has an inherent variability and is susceptibility to microorganisms. $^{1}$ In order to protect wood from both physical as well as biological weathering, application of a protective coating layer is a well-established technique for wood protection in addition to wood modification and wood preservation. The performance of a wood coating in the field or in simulated service is measured in many ways, of which micro-imaging and accompanying analysis of the visual information is a way of assessing its weathering behaviour. Techniques such as conventional light microscopy and fluorescence microscopy ${ }^{2}$ are complemented with high-end modalities as atomic force microscopy, ${ }^{3,4}$ scanning electron microscopy ${ }^{5}$ and confocal laser microscopy. ${ }^{6}$ Only the last technique can envisage a coating non destructively in three dimensions, yet, although its superior resolution, confocal microscopy still has a limited probing capacity. In addition to above-mentioned techniques, X-ray tomography is a high-end tool for fast and non-destructive threedimensional analysis. The technique has been used in several research domains for various applications ranging from plant biology ${ }^{7}$ to soil science. ${ }^{8}$ Previous studies using $\mathrm{X}$-ray tomography have been performed on thermal barrier coatings, ${ }^{9}$ paper $^{10,11}$ and cultural heritage. ${ }^{12}$ High-resolution X-ray tomography is particularly interesting for the study of wood coatings regarding the structure of the substrate and the average thickness and penetration depth of a coating. Furthermore, the digitalized coating structure allows analysis of its surface and inner structure as well. To demonstrate the power of X-ray tomography and its limitations, two coated wood samples were visualized and analyzed.

\section{Experimental Methods and Materials}

\section{Sample description}

Wood from the following tree species was used: Scots pine (Pinus silvestris) and padouk (Pterocarpus soyauxii), representing respectively a softwood and a tropical hardwood species. Pine sapwood is a light material often used as test substrate in European standards whereas padouk is a durable and dens tropical species from Africa used for the production of window joinery. The wood was cut from straightgrained material and two small boards were sawn with a growth ring angle of $45^{\circ}$ with the surface. The boards were brush-coated with an opaque solvent-borne and an opaque water-borne coating. Details of coating application and coating composition are not given, as the purpose of this paper is an illustration of X-ray imaging and accompanying analysis, not an in-depth study of wood coatings itself.

\section{Image acquisition}

Samples for scanning were prepared by sawing a section of the coated boards into parallelepiped-shaped specimen. The top section of the wood sample, measuring approximately $2 \times 2 \times 2 \mathrm{~mm}$ was scanned using the X-ray equipment built at the Centre for X-ray Tomography at Ghent University (UGCT - http://www.ugct.ugent.be). This is a state-of-the-art scanner, ${ }^{13}$ highly flexible, with in-house developed software 
for scanner control, sample reconstruction, analysis and visualization. The X-ray source, a Feinfocus nano-focus tube, can reach a focal spot size down to one $\mu \mathrm{m}$. All samples were scanned at an average voltage of $70 \mathrm{kV}$, a target current of $30 \mu \mathrm{A}$ and an exposure time of $1800 \mathrm{~ms}$ per image. A rotation step size of $0.36^{\circ}$ was used. Reconstruction took 20 min with Octopus, a server/client tomography reconstruction package for parallel and cone beam geometry (www.xraylab.com). ${ }^{14}$ With the described set-up, micron resolution can be reached, in this paper resulting in scans with voxels sizing approximately $2 \times 2 \times 2 \mu \mathrm{m}$ with $2^{16}$ greyscale levels.

111

112

113

114

115

116

\section{Image analysis}

The images were loaded in MATLAB $\AA$ for preprocessing and analysis. The aim was to quantify surface roughness, thickness and structure of the coating layer, contact surface quantification and penetration depth. The various steps of the analysis are described below.

Preprocessing. Although image quality of the reconstructed slices was satisfactory, bilateral filtering was applied as an edge-preserving smoothing technique to prevent averaging across edges and still average in smooth regions ${ }^{15}$ aiming at an improvement in image segmentation.

Segmentation. This is nearly always the most crucial part of image analysis. ${ }^{16}$ Due to the differences in X-ray density of coating, wood and noise the bilateral filtered scans could be segmented by fitting a multimodal normal distribution consisting of three Gaussian-shaped curves to the histogram of the cross-sectional slices for pine. Fig. 1 displays the histogram of a resized slice with the fit of the normal distributions for pine. The intersection between the first and second Gaussian distribution separates noise from wood. The intersection of the second and third Gaussian curve is used as threshold separating coating from wood. Clearly, the difference between wood and coating voxels is more pronounced than the demarcation between noise and wood.

\section{Fig. 1}

Cleaning. A combined morphological closing and size-based opening of the segmented volume removed isolated voxels or voxel islands, erroneously classified as coating due to imperfect segmentation.

Surface extraction. During scanning, samples were positioned upright with the coating layer parallel to the horizontal plane, yet small deviations from this horizontal position had to be corrected mathematically by rotation of the volume for correct calculation of layer thickness and penetration. Therefore, the surface of the coating layer was extracted from the segmented volume and a plane was fitted using principal component analysis. As such, the normal of the plane gives the rotation angles necessary for spatial transformation of the data.

Surface roughness. For further analysis of the extracted and rotated surface, heights were averaged to zero and several roughness parameters were computed using ImageJ with the SurfCharJ toolbox of Chinga et al.: ${ }^{17}$ arithmetical mean deviation $\left(R_{a}\right)$, root mean square deviation $\left(R_{q}\right)$, kurtosis of the assessed profile $\left(R_{k u}\right)$, skewness of the assessed profile $\left(R_{s k}\right)$, lowest valley $\left(R_{v}\right)$, highest peak $\left(R_{p}\right)$, the total height of the profile $\left(\mathrm{R}_{\mathrm{t}}\right)$. Polar angles (orientation) and azimuthal angles (direction) of the facets were also calculated based on the facet normals of the triangulated surface. 
Layer thickness and penetration depth. In order to exclude coating pores from being included erroneously in the penetration depth computation, morphological filling of pores in the coating was obligatory. Two methods were used to calculate layer thickness and penetration depth starting from the surface perpendicular downwards. Both are illustrated in Fig. $2 \mathrm{~b}$ and $2 \mathrm{c}$ with the original image in Fig. $2 \mathrm{a}$.

Fig. 2

Theoretically, penetration does not take into account the simple filling of cells that are cut open lying at the surface. Yet the extent of coating penetration is controlled by the ability to flow into open ends and by transport through interconnecting pits. ${ }^{18}$ Fig. $2 \mathrm{~b}$ represents the demarcation of the layer thickness according to method 1 following the theoretical definition. The remaining coating beneath the layer is considered to be penetrated in the substrate. Method 2, illustrated in Fig. 2c, is similar to the method elaborated in Van den Bulcke et al. ${ }^{19}$ and based on a two-dimensional view on penetration as layer thickness includes all coating material above the roughness of the substrate and penetration everything beneath it.

Porosity. Calculation of the porosity of the segmented coating volume was based upon the pore volume distribution and maximum inscribed sphere distribution.

Interface. The interface of the coating, i.e. the contact surface between coating and wood, was quantified as a measure of adhesion by computation of the area of the triangulated interface surface as an approximation of the real surface area. Logically, the fineness of the mesh determines the accuracy of the surface approximation.

All reconstructed and analyzed images of the samples were visualized with VGStudio $\operatorname{MAX}{ }^{\circledR}, \operatorname{MATLAB}{ }^{\circledR}$, ImageJ and Drishti. $^{20}$

\section{Results and Discussion}

Fig. 3 illustrates several two- and three-dimensional images representing volume renderings and analyzed data of the opaque solvent-borne and opaque water-borne coating.

\section{Fig. 3}

Fig. $3 a$ and $3 b$ are the original scans, false colour-coded and based on manual segmentation of the histogram. By virtual removal of a part of the wood substrate, the imprint of the wood surface at the bottom of the coating is clearly visible. This casting of the wood by the coating reveals, as expected, an apparently less rough print for padouk than for pine. The inserts in Fig. $3 a$ and $3 b$ are a detailed cross-sectional slice of the coated wood samples, resembling images acquired with confocal microscopy $^{6,19}$ and could be used for manual demarcation of layer thickness and penetration depth. Clearly, padouk's higher density results in a smaller contrast between coating and wood, interfering with segmentation. Fig. $3 c$ and $3 d$, with ring artefact, $^{21}$ illustrate the extracted surface of the coatings, averaged to a mean roughness of zero. Naturally, the level of detail is determined by the voxel size of the scans, which is sufficient for wood coatings. Table 1 lists several roughness parameters calculated for the two coatings under test. 
Table 1

The observation that the surface roughness of coated pine is higher than the surface roughness of padouk is self-evident as the padouk surface is in general smoother. Yet it should be stressed that these calculations, performed on a square of approximately $2 \mathrm{~mm}^{2}$ of coated padouk, are not representative for the total board due to the heterogeneity of the wood. Regions including vessels were not included in the scan but can have a significant influence on the coating's texture. By contrast, the surface roughness of pine is more or less representative for the total board surface, at least for the earlywood region as imaged in the pine scan. Typically, a waviness pattern caused by earlywood-latewood differences appears for coatings with moderate layer thickness due to incomplete levelling. An interesting view is given by the orientation and direction of the facets of the surface. Fig. 3e and $3 f$ illustrate the orientation and Fig. $3 \mathrm{~g}$ and $3 \mathrm{~h}$ direction for pine and padouk. For both coatings, orientation is low inherent to a rather flat surface with only at some specific points high angles due to a high slope. The azimuthal images more or less reflect the topography as seen in Fig. $3 \mathrm{c}$ and Fig $3 \mathrm{~d}$. Such information can be related to gloss values and can be implemented in light models such as the Beckmann theory of reflection for gloss prediction. Especially when monitoring changes during weathering, surface characterization is of high interest. ${ }^{4}$ What is more, the evolution of a small crack present in the surface of the coating indicated by the black and white arrows (Fig. 3c and 3e) could be monitored throughout weathering. In this respect, the non-destructive nature of the technique is an obvious advantage.

Determination of layer thickness and penetration depth is more complex. In order to quantify such an effect, it is necessary to be able to segment coating and wood at the interface properly and is only feasible if the greyscale levels differ significantly. The mean layer thickness and penetration depth according to method 1 are respectively $58.2 \mu \mathrm{m}$ and $3.8 \mu \mathrm{m}$ for pine and $43.8 \mu \mathrm{m}$ and $2.5 \mu \mathrm{m}$ for padouk. By definition, values of penetration are quite low. However, following method 2 as given in Van den Bulcke et al. $^{19}$ results are expected to be quite different yet the true threedimensional information is not used at its full potential with the latter definition. In this case, layer thickness and penetration depth are respectively $36.7 \mu \mathrm{m}$ and $21.4 \mu \mathrm{m}$ for pine and $37.5 \mu \mathrm{m}$ and $6.1 \mu \mathrm{m}$ for padouk. These results are more or less in agreement with the results found by Van den Bulcke et al. ${ }^{19}$ for similar coatings. The two-dimensional illustration of layer thickness and penetration according to the first method is illustrated in Fig. $4 \mathrm{a}$ and $4 \mathrm{~b}$ for pine only.

\section{Fig. 4}

These two-dimensional illustrations are sound for pine, yet for padouk misclassification results in erroneous removal of coating voxels or the addition of high-density crystals, present in the wood substrate, to the coating matrix. Although the average value smoothes such faulty segmentation and is a good estimate of layer thickness, the exact automated determination of penetration on each position is not accurate enough for padouk. Especially for automated computation of penetration, there is still work to do. To eliminate incorrect segmentation and when detailed study of penetration is aimed at, a solid solution would be differential imaging: subtraction of the images before and after coating application. Scanning with voxels $<1 \mu \mathrm{m}$ would also contribute to a better segmentation yet reduces the volume that can be scanned. 
Determination of the porosity of a coating also relates to the issue of segmentation. Mostly, pores are smaller than the voxel size of these tomography scans and are of no interest as such. However, especially when dealing with spray-coated material, these pores can be larger and can compromise coating quality. Yet careful observation reveals that sporadically, during segmentation, small parts of the coating are classified as pores although they can also be considered as low-density regions. For larger holes, this is not an issue but for smaller ones it is. For pine, the total pore volume amounts up to $0.07 \%$ of the total coating volume and for padouk this is $0.06 \%$. As an example, the pores in the pine coating are visualized in Fig. $5 \mathrm{a}$ and the pore volume distribution and radii distribution of the inscribed spheres are graphed in Fig 5b.

\section{Fig. 5}

The pore volume distribution is truncated at $1530 \mu \mathrm{m}^{3}$. Only a few larger pores are present. Although the pore volumes show a broad range, the radii of the maximum inscribed sphere are limited due to the irregular shape of most pores.

Quantification of the interface is a measure for the contact area between wood and coating and can possibly be related to adhesive strength. The area of contact for pine is $3.1 \mathrm{~mm}^{2}$ measured on a surface of $2.2 \mathrm{~mm}^{2}$ (ratio $=1.4$ ) while for padouk this is 3.2 $\mathrm{mm}^{2}$ on a surface of $2.3 \mathrm{~mm}^{2}$ (ratio $=1.4$ ). These values do not take into account the penetration of the coating. When considering penetration according to the first method, the contact area increases considerably to $6.3 \mathrm{~mm}^{2}$ (ratio $=2.7$ ) and 6.6 $\mathrm{mm}^{2}$ (ratio $=2.7$ ) respectively for pine and padouk. Ensuing, good penetration contribute to mechanical anchoring as well as chemical bridging and proper in-depth protection of the substrate.

\section{Conclusions}

High-resolution X-ray tomography is a powerful imaging modality for coating research, if only for the visual richness of the volumetric data. If proper segmentation is possible, a set of characteristics can be calculated automatically using standard image processing algorithms. First, the assessment of the surface condition is straightforward. Once extracted, any parameter can be calculated related to surface analysis and being a non-destructive technique, monitoring coating behaviour during weathering with X-ray computed tomography is an option. For wood types with a rather low density in relation to the coating, layer thickness and penetration depth can be calculated rather easily owing to a good segmentation, yet for high-density species processing should be done carefully. Nevertheless, if automated computation is unsatisfactory, one can always resort to manual measurements. Characterization of porosity, taking into account the limits of resolution, is of minor importance for brush-coated boards but can be interesting for spray-coated material. Computation of contact surface might be an indicator for adhesive strength and is easily determined once a good segmentation is obtained. All above-mentioned parameters are derived from three-dimensional scans acquired with X-ray computed tomography. Many possibilities to use these virtual data of coated wood once labelled and characterized, are available. Derivation of parameters is merely the beginning, time lapse scanning and virtual testing of performance is the next step. 
The authors wish to thank the Fund for Scientific Research-Flanders (Belgium 303 FWO) for the postdoctoral funding granted to the first author.

304

305 


\section{References}

1. Zabel, RA, Morell, JJ, Wood microbiology: decay and its prevention. Academic Press Inc., San Diego (1992)

2. de Meijer, M, Thurich, K, Militz, H, "Comparative study on penetration characteristics of modern wood coatings." Wood Sci. Technol. 32 (5) 347-365 (1998)

3. Yang, XF, Tallman, DE, Bierwagen, GP, Croll, SG , Rohlik, S, "Blistering and degradation of polyurethane coatings under different accelerated weathering tests." Polym. Degrad. Stabil. 77 (1) 103-109 (2002)

4. Yang, XF, Li, J, Croll, SG, Tallman, DE, Bierwagen, GP, "Degradation of low gloss polyurethane aircraft coatings under UV and prohesion alternating exposures." Polym. Degrad. Stabil., 80 (1) 51-58 (2003)

5. Singh, AP, Ratz, A, Dawson, BSW, "A novel method for high-resolution imaging of coating distribution within a rough-textured plywood surface." J. Coat. Technol. Res., 4 (2) 207-210 (2007)

6. Singh, AP, Dawson, BSW, "Confocal microscope - A valuable tool for examining wood-coating interface." JCT Res., 1 (3) 235-237 (2004)

7. Leroux, O, Leroux, F, Bellefroid, E, Claeys, M, Couvreur, M, Borgonie, G, Van Hoorebeke, L, Masschaele, B, Viane, R, "A new preparation method to study fresh plant structures with X-ray computed tomography." J. Microsc.-Oxf., 233 (1) 1-4 (2009)

8. Sleutel, S, Cnudde, V, Masschaele, B, Vlassenbroek, J, Dierick, M, Van Hoorebeke, L, Jacobs, P, De Neve, S, "Comparison of different nano- and microfocus X-ray computed tomography set-ups for the visualization of the soil microstructure and soil organic matter." Comput. Geosci., 34 (8) 931-938 (2008)

9. Kulkarni, A, Goland, A, Herman, H, Allen, AJ, Dobbins, T, DeCarlo, F, llavsky, J, Long, GG, Fang, S, Lawton, P, "Advanced neutron and X-ray techniques for insights into the microstructure of EB-PVD thermal barrier coatings." Mater. Sci. Eng. A-Struct. Mater. Prop. Microstruct. Process., 426 (1-2) 43-52 (2006)

10. Juuti, M, Prykari, T, Alarousu, E, Koivula, H, Myllys, M, Lahteela, A, Toivakka, M, Timonen, J, Myllyla, R, Peiponen, KE, "Detection of local specular gloss and surface roughness from black prints." Colloid Surf. A-Physicochem. Eng. Asp., 299 (1-3) 101-108 (2007)

11. Chinga-Carrasco, G, Kauko, H, Myllys, M, Timonen, J, Wang, B, Zhou, M, Fossum, JO, "New advances in the 3D characterization of mineral coating layers on paper." J. Microsc.-Oxf., 232 (2) 212-224 (2008)

12. Morigi, MP, Casali, F, Bettuzzi, M, Bianconi, D, Brancaccio, R, Cornacchia, S, Pasini, A, Rossi, A, Aldrovandi, A, Cauzzi, D, "CT investigation of two paintings on wood tables by Gentile da Fabriano." Nucl. Instrum. Methods Phys. Res. Sect. A: Accel. Spectrom. Detect. Assoc. Equip., 580 (1) 735-738 (2007)

13. Masschaele, BC, Cnudde, V, Dierick, M, Jacobs, P, Van Hoorebeke, L, Vlassenbroeck, J, "UGCT: new X-ray radiography and tomography facility." Nucl. Instrum. Methods Phys. Res. Sect. A: Accel. Spectrom. Detect. Assoc. Equip., 580 (1) 266-269 (2007)

14. Vlassenbroeck, J, Dierick, M, Masschaele, B, Cnudde, V, Van Hoorebeke, L, Jacobs, $\mathrm{P}$, "Software tools for quantification of X-ray microtomography at the UGCT." Nucl. Instrum. Methods Phys. Res. Sect. A: Accel. Spectrom. Detect. Assoc. Equip., 580 (1) 442-445 (2007) 
15. Tomasi, C, Manduchi, R, "Bilateral filtering for gray and color images." Proc. of the 1998 IEEE International Conference on Computer Vision, Bombay, India, 1998

16. Moell, M, Borgefors, G, "An image analysis method to measure cross-sectional tracheid dimensions on softwood increment cores." Wood Fiber Sci., 33 (2) 200$212(2001)$

17. Chinga, G, Johnsen, PO, Dougherty, R, Berli, EL, Walter, J, "Quantification of the 3D microstructure of SC surfaces." J. Microsc.-Oxf., 227 (3) 254-265 (2007)

18. de Meijer, "Interactions between wood and coatings with low organic solvent content.", Wageningen, the Netherlands, 1999

19. Van den Bulcke, J, Rijckaert, V, Van Acker, J, Stevens, M, "Quantitative measurement of the penetration of water-borne coatings in wood with confocal lasermicroscopy and image analysis." Holz Als Roh-und Werkst., 61 (4) 304-310 (2003)

20. Limaye, A, "Drishti - Volume Exploration and Presentation Tool", Proc. of Vis, Baltimore, USA, 2006

21. Vidal, FP, Letang, JM, Peix, G, Cloetens, P, "Investigation of artefact sources in synchrotron microtomography via virtual X-ray imaging." Nucl. Instrum. Methods Phys. Res. Sect. B-Beam Interact. Mater. Atoms, 234 (3) 333-348 (2005) 
376

\begin{tabular}{lccccccc}
\hline & $\mathrm{R}_{\mathrm{a}}$ & $\mathrm{R}_{\mathrm{q}}$ & $\mathrm{R}_{\mathrm{sk}}$ & $\mathrm{R}_{\mathrm{ku}}$ & $\mathrm{R}_{\mathrm{v}}$ & $\mathrm{R}_{\mathrm{p}}$ & $\mathrm{R}_{\mathrm{t}}$ \\
\hline Pine & 4.5 & 5.5 & -0.3 & -0.0 & -19.7 & 14.6 & 34.3 \\
Padouk & 1.2 & 1.6 & -1.3 & 11.3 & -16.6 & 4.8 & 21.4 \\
\hline
\end{tabular}




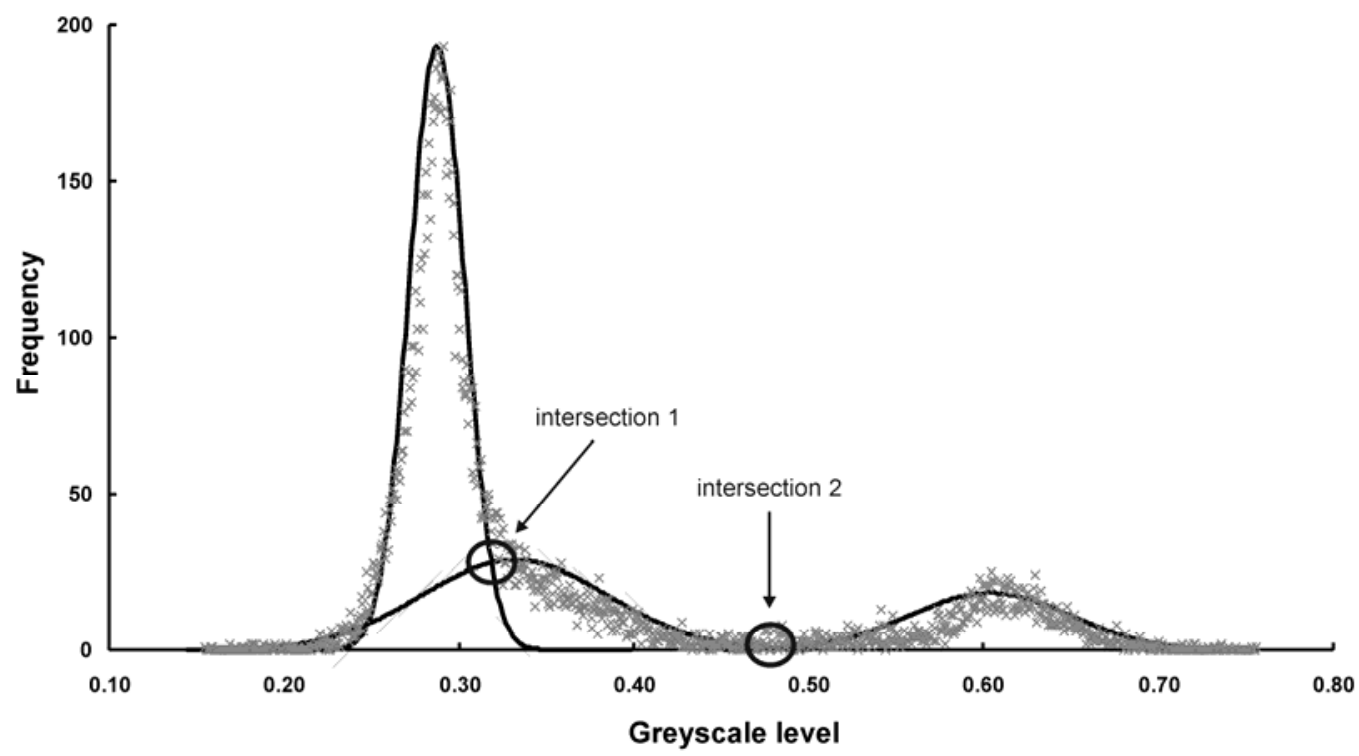

Fig. 1: Multimodal fit of three normal distributions to the histogram of a resized cross382 sectional slice of pine.

383

384

385 

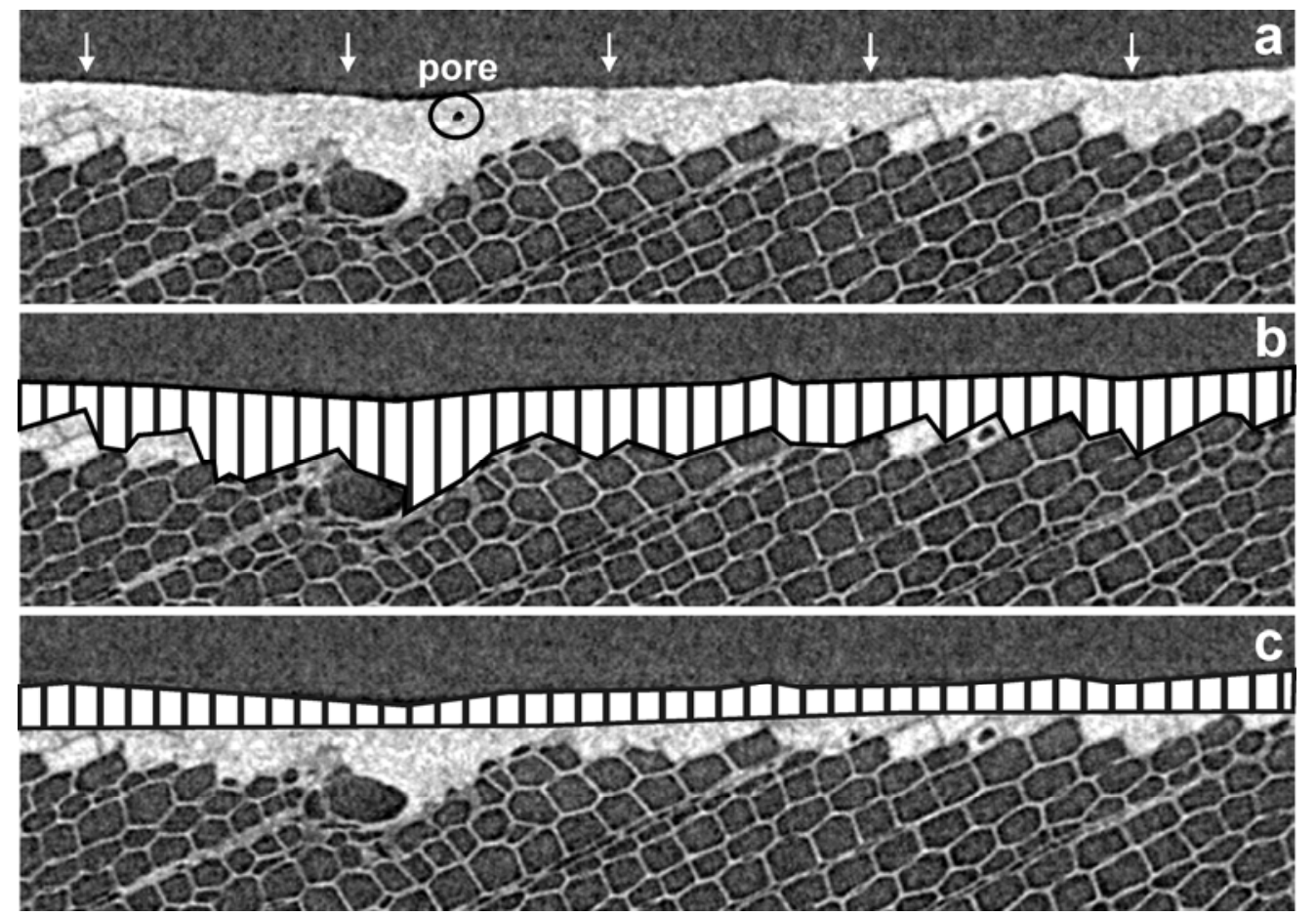

Fig. 2: (a) Original slice through the coated pine sample, (b) demarcation of layer thickness for three-dimensional penetration computation and (c) demarcation of layer thickness based on the two-dimensional approach according to Van den Bulcke et al. ${ }^{19}$ All coating material beneath the layer is considered to be penetration. 

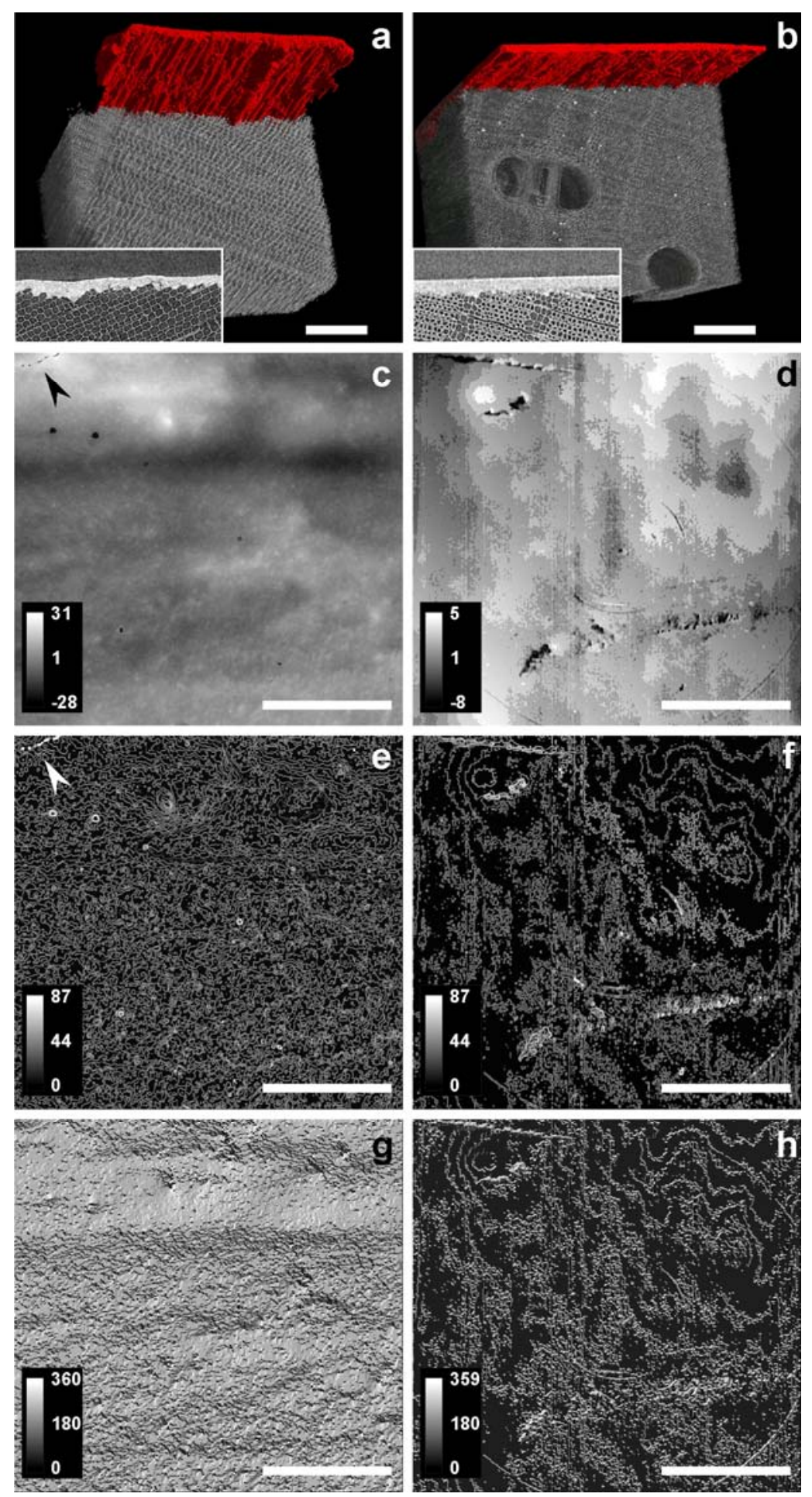

Fig. 3: (a) Original scan of Scots pine sapwood and (b) padouk with virtual removal of the wood substrate (the reader is referred to the online version for the coloured images); (c) and (d) show the surface roughness of the coating, averaged to zero with the calibration bar in $\mu \mathrm{m}$; (e) and (f) display the orientation of the facets in degrees: $0^{\circ}=$ perpendicular to surface; $(\mathrm{g})$ and $(\mathrm{h})$ represent the direction of these facets in degrees. Scale bars $=500 \mu \mathrm{m}$. 
404

405

406

407

408

409
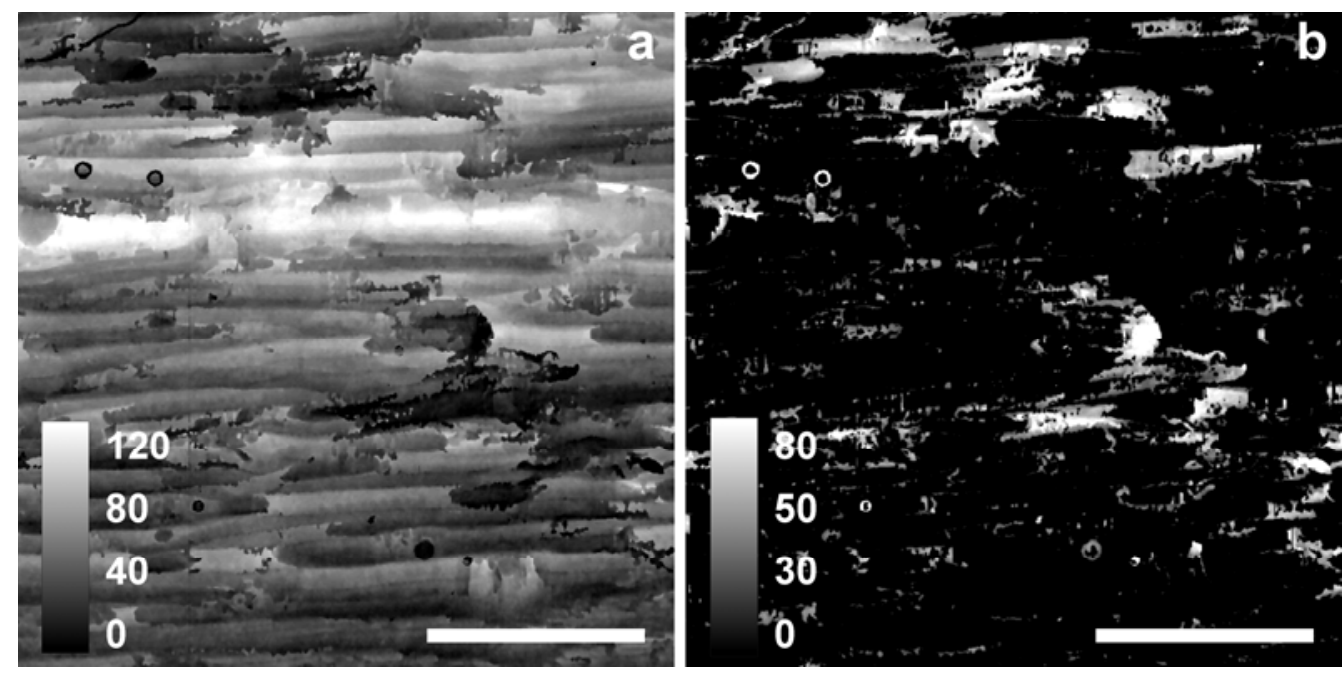

Fig. 4: (a) Layer thickness and (b) penetration depth of coated pine. Scale bars $=500$ $\mu \mathrm{m}$. 

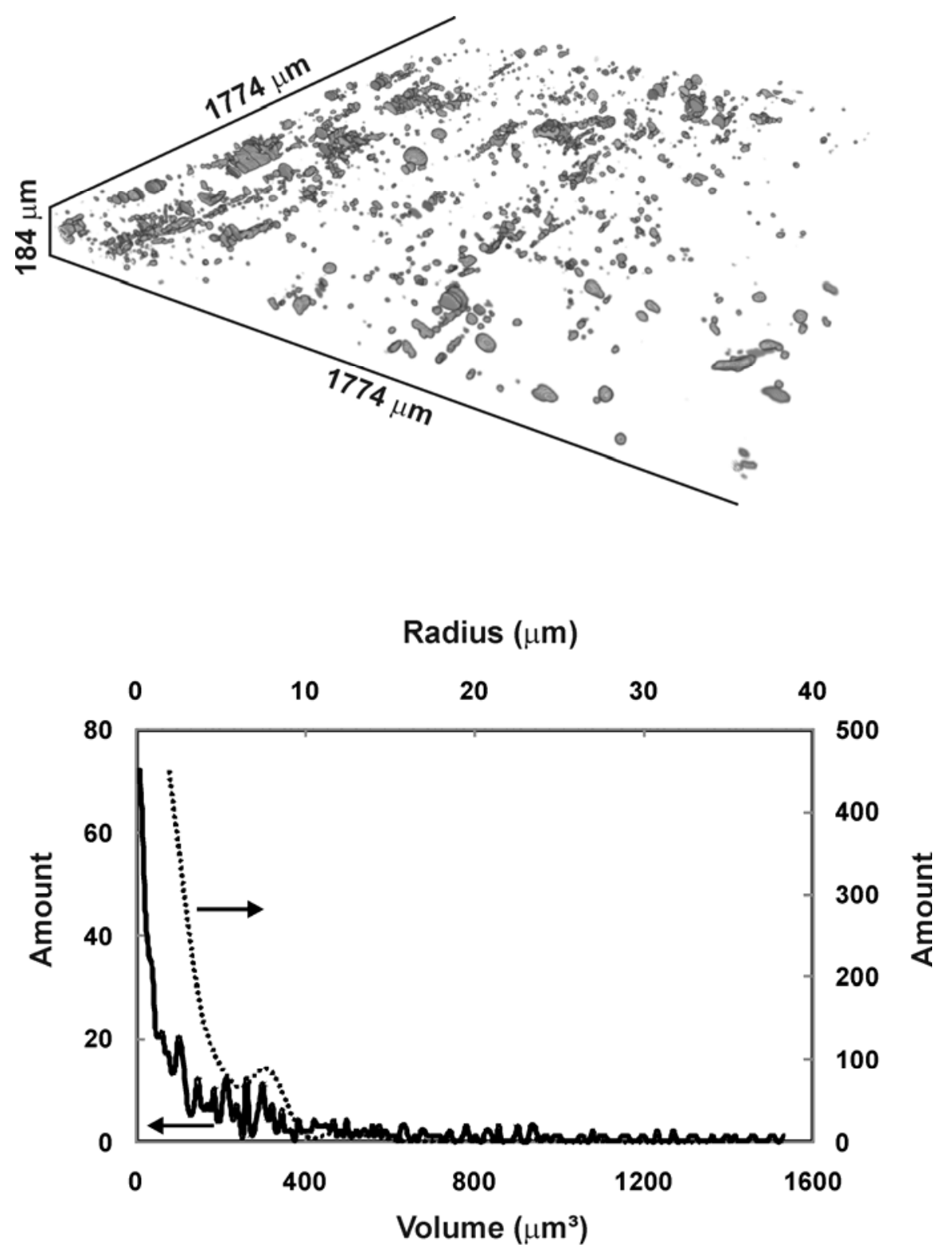

Fig. 5: (a) Three-dimensional illustration of the pores in the coating applied on the pine substrate and (b) distribution of the pore volumes (solid line) and radii of the maximum inscribed spheres (dashed line). 